\title{
FROM THE GEOMETRY OF BOX SPACES TO THE GEOMETRY AND MEASURED COUPLINGS OF GROUPS
}

\author{
KAJAL DAS
}

\begin{abstract}
In this paper, we prove that if two 'box spaces' of two residually finite groups are coarsely equivalent, then the two groups are 'uniform measured equivalent' (UME). More generally, we prove that if there is a coarse embedding of one box space into another box space, then there exists a 'uniform measured equivalent embedding' (UME-embedding) of the first group into the second one. This is a reinforcement of the easier fact that a coarse equivalence (resp. a coarse embedding) between the box spaces gives rise to a coarse equivalence (resp. a coarse embedding) between the groups.

We deduce new invariants that distinguish box spaces up to coarse embedding and coarse equivalence. In particular, we obtain that the expanders coming from $S L_{n}(\mathbb{Z})$ can not be coarsely embedded inside the expanders of $S L_{m}(\mathbb{Z})$, where $n>m$ and $n, m \geq 3$. Moreover, we obtain a countable class of residually groups which are mutually coarse-equivalent but any of their box spaces are not coarseequivalent.
\end{abstract}

Mathematics Subject Classification (2010): 20E26, 20F69, 20F65, 37A15, 37A20, 51F99.

Key terms: residually finite groups, box spaces, coarse equivalence, coarse embedding, measured equivalence, uniform measured equivalence (UME), uniform measured equivalent embedding (UME-embedding), Gromov-Hausdorff convergence, marked groups.

\section{INTRODUCTION}

A finitely generated group is called residually finite if there exists a decreasing sequence of finite index normal subgroups $\left\{G_{n}\right\}_{n \in \mathbb{N}}$ whose intersection is trivial, i.e., $G=G_{1} \unrhd G_{2} \unrhd \cdots \unrhd G_{n} \unrhd \cdots \unrhd 1$. A box space of a finitely generated residually finite group is defined as the disjoint union of $\sqcup_{n \in \mathbb{N}} G / G_{n}$. One can give a metric on the box space as follows: First, we fix a finite generating subset of $G$. This generating subset gives a metric on $G$ (which is the Cayley graph of $G$ with respect to the generating subset). Then, this metric induces a natural metric on each of $G / G_{n}$. We then assign a metric on the union such that the distance between two distinct copies $G / G_{n}$ and $G / G_{n+k}$ tends to infinity as $n \rightarrow \infty$. We refer to [22] for a rigorous description of such a metric. Sometimes the box space of a group $G$ corresponding to a sequence of normal subgroups $\left\{G_{n}\right\}_{n \in \mathbb{N}}$ as above is denoted by $\square_{G_{n}} G$. The concept of box spaces plays an important role in the context of coarse Baum-Connes conjecture and maximal Baum-Connes conjecture (see [31, [1]). Also, the expander graphs obtained from Property (T) groups (see 25]) form a significant class of box spaces.

We recall that a map $f: X \rightarrow Y$ between two metric spaces is called $\left(\rho_{+}, \rho_{-}\right)$coarse embedding if there exist two non-decreasing functions $\rho_{+}, \rho_{-}:[0, \infty) \rightarrow$ $[0, \infty)$ tending to $+\infty$ such that $\rho_{-}\left(d_{X}\left(x_{1}, x_{2}\right)\right) \leq d_{Y}\left(f\left(x_{1}\right), f\left(x_{2}\right)\right) \leq \rho_{+}\left(d_{X}\left(x_{1}, x_{2}\right)\right)$ for every $x_{1}, x_{2} \in X$; it is called a $\left(\rho_{+}, \rho_{-}, c\right)$-coarse equivalence if moreover $Y$ is the $c$-neighbourhood of $f(X)$ for some $c \geq 0$. The study of coarse embedding of a metric space into another metric space mainly started from the the proof of 'coarse Baum-Connes conjecture' by Yu in 31] for every discrete metric space with 
'bounded geometry' which are coarsely embeddable inside a Hilbert space and this result implies the Novikov conjecture for all closed manifolds whose fundamental group with the word-length metric with respect to some generating subset coarsely embeds into a Hilbert space. We should mention that Yu's idea was implicit in Gromov's intution of approaching 'Novikov's conjecture' in 16. Yu also introduced 'Property A' for metric spaces with bounded geometry and showed that the spaces with Property A coarsely embed into a Hilbert space. This class of metric spaces includes Gromov's word hyperbolic groups, discrete subgroups of connected Lie groups and amenable groups. It was once conjectured that any 'bounded geometry uniformly discrete' metric space can be coarsely embedded inside a Hilbert space. Then, Gromov came up with a counter-example and proved that expanders can not be coarsely embedded inside a Hilbert space [18, which follows immediately from an inequality of Matoušek [26].

There is a strong interaction between the analytic properties of residually finite groups and the coarse-geometric properties of the corresponding box spaces. Let $G$ be a residually finite group and $\left\{G_{n}\right\}_{n \in \mathbb{N}}$ be a sequence of normal subgroups as above. Here is a general picture on the interactions between the residually finite groups and their box spaces.

1.

$$
G \text { is amenable } \longleftrightarrow \square_{G_{i}} G \text { has Property } A
$$

2.

$G$ has Haggerup $\leftrightarrow \square_{G_{i}} G$ has a coarse fibred embedding into a Hilbert space

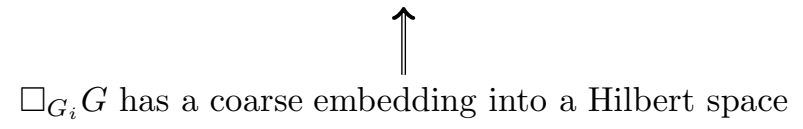

3.

G has Property $\tau$ w.r.t. $\left\{G_{i}\right\}_{i \in \mathbb{N}} \Longleftrightarrow \square_{G_{i}} G$ is an expander

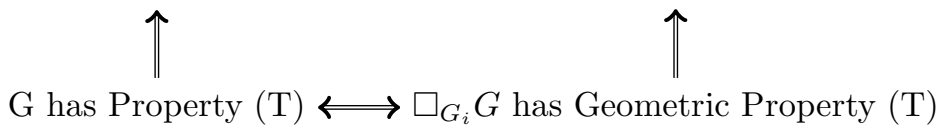

We refer the readers to [10, 30], 24], 25] for the above implications. However, Arzhantseva, Guentner and Spakula [3] give the first example of a metric space (with bounded geometry) without Yu's Property A which coarsely embeds into a Hilbert space. This is constructed as a box space of $F_{2}$, the free group with two generators. Since any expander sequence can not be coarsely embedded inside a Hilbert space, Gromov asked the following natural question: Given a metric space with bounded geometry which does not embed coarsely into a Hilbert space, does it necessarily contain a 'weakly embedded' expander? Recently, Arzhantseva and Tessera in [4] answer this question negatively. This counter example also comes as a box space of a finitely generated residually finite group. We should remark that two box spaces of a group can have different coarse-geometric properties. For example, by Selberg's theorem (see 24) there exists a box space of $F_{2}$ which is an expander. On the other hand, using [3], we can construct another chain of normal subgroups of $F_{2}$ so that the corresponding box space coarsely embeds into a Hilbert space. Therefore, these two box spaces are not coarsely equivalent.

Our motivation in this article is to find obstructions for two box spaces to be coarsely equivalent (or to coarsely embed into one another). It is not very difficult to prove that if there is a coarse equivalence (resp. coarse embedding) between two box spaces, then there is a coarse equivalence (resp. coarse embedding) between the 
corresponding groups. We have proved this result in Section 2 (see 3.1). This result also appears in a general form for 'marked groups' in [23. We push this result one step further. We prove that if two box spaces are coarsely equivalent, then the corresponding groups are 'uniform measured equivalent'(UME). More generally, we prove that if there is a coarse embedding of a box space into another box space, then there is a 'UME-embedding' of the first group into the second one. Uniform Measured Equivalence is a sub-equivalence relation of 'Measure Equivalence' on finitely generated groups introduced by Shalom in [29]. Two countable discrete groups $\Gamma$ and $\Lambda$ are called Measured Equivalent(ME) if they have commuting measure preserving free actions on a Borel space $(X, \mu)$ with finite measure Borel fundamental domains, say $X_{\Gamma}$ and $X_{\Lambda}$ respectively. The space $(X, \mu)$ is called a 'measured coupling space' for the groups $\Gamma$ and $\Lambda$. If, moreover, the action of an element of one group, say $\Gamma$, on the fundamental domain of another group, say $X_{\Lambda}$, is covered by finitely many $\Lambda$-translates of $X_{\Lambda}$, then these two groups are called UME. We refer the readers to Subsection 2.1 for the definition of UME-embedding. Now, we formally state the main theorem of our paper.

Theorem 1.1. Suppose $G$ and $H$ are two finitely generated residually finite groups with two decreasing sequences of finite index normal subgroups $1 \unlhd \cdots \unlhd G_{n} \unlhd$ $\cdots \unlhd G_{1}=G$ and $1 \unlhd \cdots \unlhd H_{n} \unlhd \cdots \unlhd H_{1}=H$. If the box space of $G$ with respect to $\left\{G_{n}\right\}_{n \in \mathbb{N}}$, denoted by $\square_{G_{n}} G$, and the box space of $H$ with respect to $\left\{H_{n}\right\}_{n \in \mathbb{N}}$, denoted by $\square_{H_{n}} H$ are coarsely equivalent, then $G$ and $H$ are UME. Similarly, we prove that if there is a coarse embedding of $\square_{G_{n}} G$ into $\square_{H_{n}} H$, then there exists a 'UME-embedding' of $G$ into $H$.

As a consequence of the above theorem, we obtain the following invariants that distinguish box spaces up to coarse embedding and coarse equivalence.

Corollary 1.2. Suppose there is a coarse embedding of a box space of $G$ into a box space of $H$ as above. Then,

(i) if there exists a metrically proper affine action of $H$ on an $L^{p}$-space, then there exists a metrically proper affine action of $G$ on an $L^{p}$ space as well, where $1 \leq p \leq \infty$;

(ii) the $R$-cohomological dimension of $G$ is less than equal to the $R$-cohomological dimension of $H$, we denote it by $c d_{R} G \leq c d_{R} H$, where $R$ is a commutative ring containing $\mathbb{Q}$.

By a deep result of Gaboriau ([15], Théorème 6.3), if two countable groups $\Gamma$ and $\Lambda$ are measured equivalent, then $\beta_{i}^{(2)}(\Gamma)=c \beta_{i}^{(2)}(\Lambda)$ for all $i \in \mathbb{N}$, where $c$ is a constant independent of $i$. Therefore, using Theorem 1.1 we obtain the following corollary.

Corollary 1.3. If two box spaces of $G$ and $H$ are coarsely equivalent, then the $l^{2}$-betti numbers of $G$ and $H$ must be proportional.

A special case of this corollary can be found in [14] (see Proposition 2.1 and Proposition 3.4).

1.1. Acknowledgements. I am very grateful to my adviser Romain Tessera for suggesting me this problem (Theorem 1.1) and helping me in the course of proving the theorem. I am indebted to Ana Khukhro and Alain Valette for inviting me at University of Neuchatel and having an illuminating discussion on a similar project they were working on. I would also like to thank Damien Gaboriau for some very useful discussions and Damian Sawicki for pointing out some of the typos.

1.2. Organization. In Section 2, we introduce our necessary definitions, notations and abreviations. In Section 3, we prove that if there is a coarse equivalence between 
two box spaces, then the corresponding groups are coarsely equivalent. We prove our main theorem 1.1 in Section 4. We divide this section into three subsections: In Subsection 4.1 we construct a 'topological coupling space' for the groups; in Subsection 4.2 we give a non-zero measure on the topological coupling space which is invariant under the actions of both groups, i.e., we make the topological coupling space into a measured coupling space; finally in Subsection 4.2 we prove our main theorem [1.1. In Section 5, we discuss some applications of Proposition 3.1 and Theorem 1.1.

\section{Preliminaries: some Definitions, notations AND ABREViations:}

2.1. In [16], Gromov first formulates a topological criterion for quasi-isometry and introduces measured equivalence as a measure theoretic counterpart of quasiisometry. In [29], Shalom slightly modifies the 'topological coupling space' constructed by Gromov and also gives a topological criterion for coarse embedding. We will mainly follow Shalom's construction of 'topological coupling' in our proof. For countable groups $\Lambda$ and $\Gamma$, there exists a coarse embedding $\phi: \Lambda \rightarrow \Gamma$ if and only if there exists a locally compact space $X$ on which both $\Lambda$ and $\Gamma$ act properly and continuously with a compact-open fundamental domain $X_{\Gamma}$ of $\Gamma$ in $X$ and the actions of $\Gamma$ and $\Lambda$ commute. Replacing $\Gamma$ with a direct product $\Gamma \times M$ for some finite group $M$, we can assume that there exists a compact-open fundamental domain $X_{\Gamma}$ for $\Gamma$ satisfying $X_{\Gamma} \subseteq X_{\Lambda}$, where $X_{\Lambda}$ is a Borel fundamental domain of $\Lambda$. Moreover, $\phi$ will be a coarse equivalence between $\Gamma$ and $\Lambda$ if and only if after replacing $\Gamma$ with a direct product $\Gamma \times M$ for some finite group $\mathrm{M}$, there exists a topological space $X$ with the following three properties: (i) both $\Lambda$ and $\Gamma$ act continuously, properly and freely on $X$; (ii) there exist fundamental domains $X_{\Gamma}$ and $X_{\Lambda}$, for $\Gamma$ and $\Lambda$ respectively, which are compact and open; (iii) $X_{\Gamma} \subseteq X_{\Lambda}$ ([29], Theorem 2.1.2, p. 129). The space $X$ is called a topological coupling space for $\Gamma$ and $\Lambda$.

In a similar way, as UME is defined in the previous section, we define uniform measured equivalence embedding (UME-embedding) as follows: We say that there is a UME-embedding of $\Lambda$ inside $\Gamma$ if there exists a Borel space $X$ with a non-zero mesure $\mu$ and measure preserving free commuting actions of $\Lambda$ and $\Gamma$ on $X$ such that there exists a finite measure fundamental domain $X_{\Gamma}$ of $\Gamma$ in $X$ and the action on $X_{\Gamma}$ by an element $\lambda$ from $\Lambda$ can be covered by finitely many $\Gamma$-translates of $X_{\Gamma}$ (the measure of $X_{\Lambda}$ may not necessarily be finite or the action on $X_{\Lambda}$ by an element $\gamma$ of $\Gamma$ may not necessarily be covered by finitely many $\lambda$-translates of $X_{\Lambda}$ ).

Given an ME-coupling $(X, \mu)$ between $\Gamma$ and $\Lambda$, with $X_{\Gamma}$ and $X_{\Lambda}$ being the fundamental domains of $\Gamma$ and $\Lambda$ respectively, we define the cocyle $\alpha: \Gamma \times X_{\Lambda} \rightarrow \Lambda$ (resp. $\beta: \Lambda \times X_{\Gamma} \rightarrow \Gamma$ ) by the rule: for all $x \in X_{\Lambda}$, and all $\gamma \in \Gamma, \alpha(\gamma, x) \gamma x \in X_{\Lambda}$ (and symmetrically for $\beta$ ).

2.2. In this article, we denote the finite generating subset of $G$ by $S_{G}$, where $S_{G}$ is symmetric, i.e., $S_{G}=S_{G}^{-1}$. The metric defined by $S_{G}$ on $G$ will be denoted by $d_{G}$. We use $d_{G / G_{n}}$ for the induced metric on $G / G_{n}$. We denote the identity elements of $G$ and $G / G_{n}$ by $1_{G}$ and $1_{G / G_{n}}$, respectively. Sometimes, when the group is clear from the context, we use 1 for the identity element of the group. The ball of radius $r$ around an element $g$ in $G$ will be denoted by $B_{r}^{G}(g)$. We use similar type of notations for $H$ and $H / H_{n}$ as well. 
2.3. It is easy to observe that if we have a $\left(\rho_{+}, \rho_{-}, c\right)$-coarse equivalence (resp. ( $\rho_{+}, \rho_{-}$)-coarse embedding) of $\square_{G_{n}} G$ into $\square_{H_{n}} H$, then passing to a subsequence and reindexing the components, both for $\square_{G_{n}} G$ and $\square_{H_{n}} H$, we can assume that there is a $\left(\rho_{+}, \rho_{-}, c\right)$-coarse equivalence (resp. $\left(\rho_{+}, \rho_{-}\right)$-coarse embedding) $f_{n}:=\left.f\right|_{G / G_{n}}$ from $G / G_{n}$ to $H / H_{n}$ for all $n$. Moreover, we can assume that $f\left(1_{G / G_{n}}\right)=1_{H / H_{n}}$ for all $n$. We should remark that for finitely generated groups coarse equivalence coincides with quasi-isometry 19 .

2.4. We now define Gromov-Hausdorff convergence (GH convergence) of compact metric spaces. We first need to define some terms before going into the definition of GH convergence. Suppose $X$ and $Y$ are two metric spaces and $f: X \rightarrow Y$ is a map. We define the 'distortion' of $f$ by the following quantity:

$$
\operatorname{disf}:=\sup _{\left\{x_{1}, x_{2}\right\}}\left|d_{Y}\left(f\left(x_{1}\right), f\left(x_{2}\right)\right)-d_{X}\left(x_{1}, x_{2}\right)\right| .
$$

$f: X \rightarrow Y$ is called an $\epsilon$-isometry for some $\epsilon \geq 0$ if disf $\leq \epsilon$ and $Y$ is an $\epsilon$ neighborhood of $f(X)$. We sometimes say that ' $f(X)$ is $\epsilon$-dense in $Y$ ' if $Y$ is an $\epsilon$-neighborhood of $f(X)$. There are several equivalent formulations of GromovHausdorff convergence of compact metric spaces, we choose the following one for our purpose (see [5, p. 260): A sequence $\left\{X_{n}\right\}$ of compact metric spaces converges to a compact metric space $X$ if there is a sequence $\left\{\epsilon_{n}\right\}_{n \in \mathbb{N}}$ of positive numbers and a sequence of maps $f_{n}: X_{n} \rightarrow X$ such that every $f_{n}$ is an $\epsilon_{n}$-isometry and $\epsilon_{n} \rightarrow 0$ as $n \rightarrow \infty$.

\section{From COARSE EQUivalence of TWO BoX SPACES TO THE COARSE EQUIVALENCE OF THEIR CORRESPONDING GROUPS}

Proposition 3.1. If $\square_{G_{n}} G$ and $\square_{H_{n}} H$ are coarsely equivalent, then $G$ and $H$ are also coarsely equivalent. Similarly, if there is a coarse embedding of the box space $\square_{G_{n}} G$ into the box space $\square_{H_{n}} H$, then there exists a coarse embedding of $G$ into $H$.

Proof. We give the proof of the first part of this proposition. The second part can be proved in a similar way. Let $\square_{G_{n}} G$ and $\square_{H_{n}} H$ be coarsely equivalent. From the discussion in Subsection 2.3. we can assume that there exists a $\left(\rho_{+}, \rho_{-}, c\right)$-coarse equivalence $\phi_{n}: G / G_{n} \rightarrow H / H_{n}$ with $\phi_{n}\left(1_{G / G_{n}}\right)=1_{H / H_{n}}$ for all $n$.

For each ball $B_{r}^{G}(1)$ in $G$, we will construct a map $f_{r}$ from $B_{r}^{G}(1)$ into $B_{\rho_{+}(r)}^{H}(1)$ in $H$ so that they are compatible in the sense $\left.f_{r}\right|_{B_{r-1}^{G}(1)}=f_{r-1}$ for all $r \in \mathbb{N} \backslash\{1\}$. The construction is as follows:

For each ball of radius $r$ in $G$, there exists an integer $m_{r}$ such that the projection from $G$ onto $G / G_{m_{r}}$ is injective on $B_{r}^{G}(1)$. Since $\phi_{m_{r}}$ is a $\left(\rho_{+}, \rho_{-}, c\right)$-coarse equivalence, the image $\phi_{m_{r}}\left[B_{r}^{G / G_{m_{r}}}(1)\right]$ is inside $B_{\rho_{+}(r)}^{H / H_{m_{r}}}(1)$. We also find a large integer $k_{r}$ such that the projection $H \rightarrow H / H_{k_{r}}$ is injective on $B_{\rho_{+}(r)}^{H}(1)$. Without loss of generality, we assume that $l_{r}:=m_{r}=k_{r}$. We define $\widetilde{\phi}_{l_{r}}: B_{r}^{G}(1) \rightarrow B_{\rho_{+}(r)}^{H}(1)$ so that the following diagram commutes:

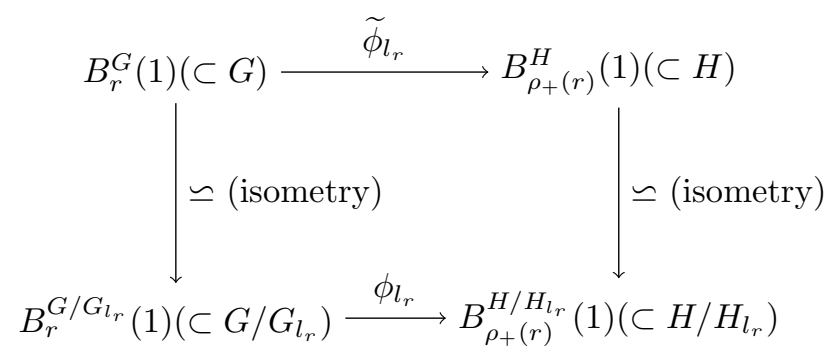


Now, we consider the ball $B_{1}^{G}\left(1_{G}\right)$. If we restrict each of $\left\{\widetilde{\phi}_{l_{r}}\right\}_{r \in \mathbb{N}}$ on $B_{1}^{G}\left(1_{G}\right)$, there exists a subsequence of $\left\{\widetilde{\phi}_{l_{r}}\right\}_{r \in \mathbb{N}}$ so that each of the functions in the subsequence coincides on $B_{1}^{G}\left(1_{G}\right)$. We take this subsequence and restrict each of the functions in this subsequence on $B_{2}^{G}\left(1_{G}\right)$. As before, we again extract another subsequence from $\left\{\widetilde{\phi}_{l_{r}}\right\}_{r \in \mathbb{N}}$ so that the functions in the subsequence agree on $B_{2}^{G}\left(1_{G}\right)$. In this way, using induction on the radius $r$ of the ball $B_{r}^{G}\left(1_{G}\right)$ and applying 'Cantor's diagonal argument', we obtain $f_{r}: B_{r}^{G}(1)(\subseteq G) \rightarrow B_{\rho_{+}(r)}^{H}(1)(\subseteq H)$ such that $\left.f_{r}\right|_{B_{r-1}^{G}(1)}=f_{r-1}$ for all $r \in \mathbb{N} \backslash\{1\}$. Now, taking the limit of $f_{r}$ as $r \rightarrow \infty$, we construct a $\left(\rho_{+}, \rho_{-}, c\right)$-coarse equivalence $\phi: G \rightarrow H$ with $\phi\left(1_{G}\right)=1_{H}$ such that there exists a subsequence $\left\{n_{r}\right\}_{r \in \mathbb{N}}$ satisfying the following diagram:

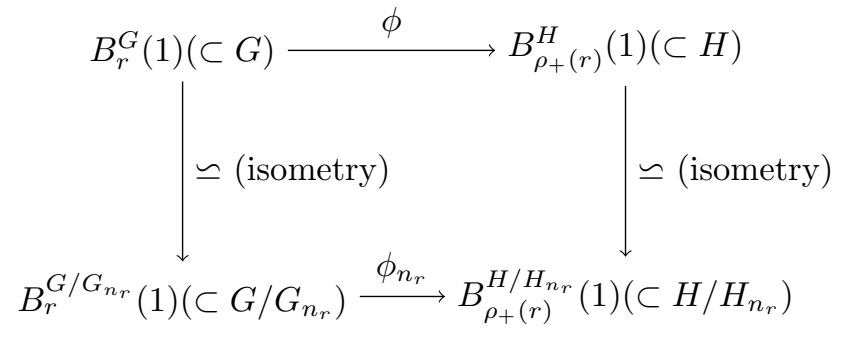

Remark 3.2. In the abovementioned construction of $\phi$ from $\left\{\phi_{n}\right\}_{n \in \mathbb{N}}$, after passing through the subsequnce $\left\{n_{r}\right\}_{r \in \mathbb{N}}$ and reindexing it, we obtain that

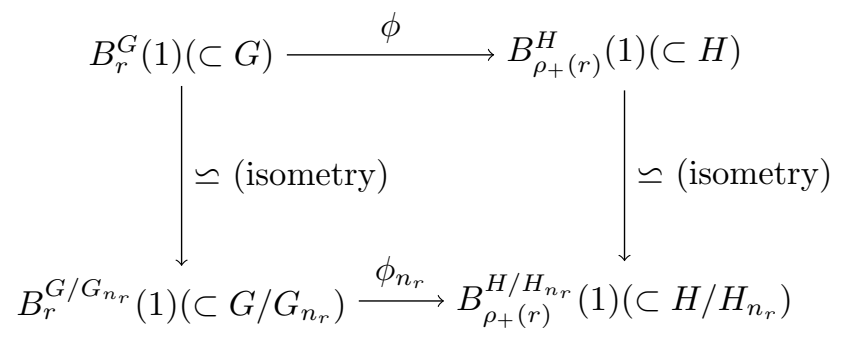

We denote this construction of $\phi$ from $\left\{\phi_{n}\right\}_{n \in \mathbb{N}}$ by the notation $\left\{\phi_{n}\right\}_{n \in \mathbb{N}} \stackrel{\left\{n_{r}\right\}_{r \in \mathbb{N}}}{\longrightarrow} \phi$.

\section{From coarse equivalence of two box spaces to UME of their CORRESPONDING GROUPS}

4.1. The construction of the topological coupling. We suppose that there is a $\left(\rho_{+}, \rho_{-}, c\right)$-coarse equivalence $\phi$ between $\square_{G_{n}} G$ to $\square_{H_{n}} H$. As discussed in 2.3 we assume that $\phi$ maps $G / G_{n}$ into $H / H_{n}$, the image $\phi\left[G / G_{n}\right]$ is $c$-dense in $H / H_{n}$ and $\phi\left(1_{G / G_{n}}\right)=1_{H / H_{n}}$ for all $n$.

We first recall the 'topological coupling space' described in [20] and 29]. We define $W=\left\{f: G \rightarrow H \mid f\right.$ is a $\left(\rho_{+}, \rho_{-}, c\right)$ - coarse equivalence $\}$. By Proposition 3.1. $W$ is non-empty. We consider the set of all functions from $G$ to $H$ with pointwise convergence topology or with the topology of compact convergence. We induce the subspace topology to $W$. Since the set of all functions from $G$ to $H$ with pointwise convergence topology is a regular space with countable basis, by 'Uryshon metrization theorem' it is metrizable [28. We, in particular, give the following metric on $W$ :

$$
d(\phi, \psi)=2^{-\sup \left\{\left.r|\phi|_{B_{r}\left(1_{G}\right)} \equiv \psi\right|_{B_{r}\left(1_{G}\right)}\right\} .}
$$

It is easy to see that the topology induced by this metric coincides with the pointwise convergence topology on $W$ and $W$ is a closed subset of the set of all functions from 
$G$ to $H$ with the pointwise convergence topology. Moreover, $W$ is a locally compact space [20. We recall the argument briefly. Fix an element $\phi_{0} \in W$. We consider the neighborhood $\mathcal{U}_{(F, k)}:=\left\{\phi \in W \mid d_{G}\left(\phi(x), \phi_{0}(x)\right) \leq k\right.$ for all $\left.x \in F\right\}$ of $\phi_{0}$ corresponding to a non-empty finite subset $F$ in $G$ and a number $k>0$. Now, by Ascoli's theorem, $\mathcal{U}_{(F, k)}$ is a compact neighborhood of $\phi_{0}$.

We define $G$ and $H$ (left) actions on a function $\phi$ in $W$ as follows:

$$
g h \phi(x)=h \phi\left(g^{-1} x\right)
$$

Let $Y:=\left\{\phi: G \rightarrow H \mid \phi \in W\right.$ and $\left.\phi\left(1_{G}\right)=1_{H}\right\}$. Again using Proposition 3.1, we know that $Y$ is non-empty. We induce the metric of $W$ on $Y$. We define $G$ action on $Y$ by

$$
[g \cdot \phi]\left(g^{\prime}\right)=\left[\phi\left(g^{-1}\right)\right]^{-1} \phi\left(g^{-1} g^{\prime}\right) .
$$

It is easy to see that $G$ actions on $W$ and $Y$ are continuous. By [20] (p. 99), we know that $W$ is a 'topological coupling space' of $G$ and $H$, where $Y$ is a compact fundamental domain of $H$.

If there is a $\left(\rho_{+}, \rho_{-}, c\right)$-coarse equivalence (resp. $\left(\rho_{+}, \rho_{-}\right)$-coarse embedding) $\phi_{n}: G / G_{n} \rightarrow H / H_{n}$ for all $n$, then there exists a finite group $M$, independent of $n$, such that there is an injective coarse equivalence (resp. coarse embedding) $\widetilde{\phi_{n}}: G / G_{n} \rightarrow H / H_{n} \times M$ for all $n$ with some modification of $\left(\rho_{+}, \rho_{-}, c\right)$ (resp. $\left.\left(\rho_{+}, \rho_{-}\right)\right)$, which we denote by the same notation as before. We define $Z_{n}:=\{f$ : $G / G_{n} \rightarrow H / H_{n} \times M \mid f$ is injective and $f$ is a $\left(\rho_{+}, \rho_{-}, c\right)-$ coarse equivalence $\}$ and $X_{n}:=\left\{f \in Z_{n} \mid f\left(1_{G / G_{n}}\right)=1_{H / H_{n}}\right\}$. In a similar way, as defined on $W$, we give a metric $d_{n}$ on each $Z_{n}$ and a $G$-action on it (through the action of $G / G_{n}$ ). By the construction given in 3.1, for a sequence of injective $\left(\rho_{+}, \rho_{-}, c\right)$-coarse equivalence (resp. injective $\left(\rho_{+}, \rho_{-}\right)$-coarse embedding) $f_{n}: G / G_{n} \rightarrow H / H_{n} \times M$ for all $n$, we will have an injective $\left(\rho_{+}, \rho_{-}, c\right)$-coarse equivalence (resp. injective $\left(\rho_{+}, \rho_{-}\right)$-coarse embedding) $\tilde{f}$ from $G$ into $H \times M$. For the notational convenience, in the remaining part of this subsection and in Subsection 4.2 we will replace $H_{n} \times M$ by $H_{n}$ and $H \times M$ by $H$.

For our purpose, we will construct another topological coupling $Z(\subseteq W)$ for $G$ and $H$. We will take the Gromov-Haudorff limit of the collection of finite metric spaces $X_{n}$ (possibly after passing to a subsequence) and identify the limit with a $G$-invariant compact subset $X$ of $Y$ (Proposition 4.1), which will turn out to be a new fundamental domain for $H$, and we will define the new 'topological coupling space' as $Z=H X$.

Proposition 4.1. There exists a subsequence of $\left\{X_{n}\right\}_{n \in \mathbb{N}}$ which converges to a $G$-invariant compact subspace $X$ of $Y$ in Gromov-Hausdorff topology.

We refer the reader to 2.4 for the definition of Gromov-Hausdorff topology. Before going into the proof of 4.1 we prove the following lemmas.

Lemma 4.2. The sequence $\left\{X_{n}\right\}_{n \in \mathbb{N}}$ of compact metric spaces is 'uniformly totally bounded', i.e.,

- there is a constant $D$ such that diam $X_{n} \leq D$ for all $n$;

- for all $\epsilon>0$ there exists a natural number $N=N(\epsilon)$ such that every $X_{n}$ contains an $\epsilon$-net consisting of at most $N$ points.

Sometimes, this type of sequence $\left\{X_{n}\right\}_{n \in \mathbb{N}}$ is also called 'relatively compact' in Gromov-Hausdorff metric. 
Proof. Fix $\epsilon>0$. We choose an arbitrarily large integer $R$ such that $2^{-R}<\epsilon$. Since diam $X_{n} \leq 1$ for all $n$, the first criterion is trivially satisfied. We now prove the second criterion. We construct a map

$h_{n}: X_{n} \rightarrow \mathcal{F}\left(B_{R}^{G / G_{n}}\left(1_{G / G_{n}}\right), B_{\rho_{+}(R)}^{H / H_{n}}\left(1_{H / H_{n}}\right)\right) \quad$ defined by $\left.\xi \mapsto \xi\right|_{B_{R}^{G / G_{n}}\left(1_{G / G_{n}}\right)}$, where $\mathcal{F}\left(B_{R}^{G / G_{n}}\left(1_{G / G_{n}}\right), B_{\rho_{+}(R)}^{H / H_{n}}\left(1_{H / H_{n}}\right)\right)$ is the collection of all maps from the ball of radius $R$ in $G / G_{n}$ around $1_{G / G_{n}}$, denoted by $B_{R}^{G / G_{n}}\left(1_{G / G_{n}}\right)$, to the ball of radius $\rho_{+}(R)$ in $H / H_{n}$ around $1_{H / H_{n}}$, denoted by $B_{\rho_{+}(R)}^{H / H_{n}}\left(1_{H / H_{n}}\right)$. The map is well-defined because the elements of $X_{n}$ are $\left(\rho_{+}, \rho_{-}, c\right)$-coarse equivalence. For all $X_{n}$, we can choose a subset $A_{n}$ of $X_{n}$ such that the restriction of $h_{n}$ on $A_{n}$ is injective and the image of $A_{n}$ under $h_{n}$ covers the whole image of $X_{n}$.

We claim that $A_{n}$ is an $\epsilon$-net in $X_{n}$. Take any $\xi$ in $X_{n}$. By the definition of $A_{n}$, there exists an element $\eta \in A_{n}$ so that $\xi$ and $\eta$ belong to the same fiber of the map $h_{n}$. Therefore, $\xi$ and $\eta$ coincides on the ball $B_{R}^{G / G_{n}}\left(1_{G}\right)$, which implies that $d_{X}(\xi, \eta) \leq 2^{-R}<\epsilon$. Hence, $A_{n}$ is an $\epsilon$-net in $X_{n}$.

Now, we compute an estimate of the cardinality of the set $A_{n}$. We observe that

$$
\begin{aligned}
\left|\mathcal{F}\left(B_{R}^{G / G_{n}}\left(1_{G / G_{n}}\right), B_{\rho_{+}(R)}^{H / H_{n}}\left(1_{H / H_{n}}\right)\right)\right| & \leq\left|B_{R}^{G / G_{n}}\left(1_{G / G_{n}}\right)\right|\left|B_{\rho_{+}(R)}^{H / H_{n}}\left(1_{H / H_{n}}\right)\right| \\
& \leq\left|S_{G}\right|^{R}\left|S_{H}\right|^{\rho_{+}(R)},
\end{aligned}
$$

where $S_{G}$ and $S_{H}$ are two generating subsets of $G$ and $H$ respectively. Therefore, $\left|A_{n}\right| \leq\left|S_{G}\right|^{R}\left|S_{H}\right|^{\rho_{+}(R)}$ for all $n$. Hence, $\left\{X_{n}\right\}_{n \in \mathbb{N}}$ is uniformly totally bounded.

Lemma 4.3 .

$$
\left\{\phi_{n}\right\}_{n \in \mathbb{N}} \stackrel{\left\{l_{r}\right\}_{r \in \mathbb{N}}}{\longrightarrow} \phi \Rightarrow\left\{g \cdot \phi_{n}\right\}_{n \in \mathbb{N}} \stackrel{\left\{l_{m_{r}}\right\}_{r \in \mathbb{N}}}{\longrightarrow} g \cdot \phi
$$

where $\phi_{n} \in X_{n}, \phi \in X$ and $m_{r}=\max \left\{r+\left|g^{-1}\right|,\left\lceil\rho_{+}\left(r+\left|g^{-1}\right|\right)\right\rceil\right\}$. For the notation $\left\{\phi_{n}\right\}_{n \in \mathbb{N}} \stackrel{\left\{l_{r}\right\}_{r \in \mathbb{N}}}{\longrightarrow} \phi$, we refer the reader to Remark 3.2.

Proof. First, we recall the definition of $g \cdot \phi_{n}$ and $g \cdot \phi$, where $\phi_{n} \in X_{n}$ and $\phi \in X$. They are defined as follows:

$$
\left[g \cdot \phi_{n}\right](\bar{x})=\left[\phi_{n}\left(g^{-1}\right)\right]^{-1} \phi_{n}\left(g^{-1} \bar{x}\right) \text { and }[g \cdot \phi](x)=\left[\phi\left(g^{-1}\right)\right]^{-1} \phi\left(g^{-1} x\right),
$$

where $\bar{x} \in G / G_{n}$ and $x \in G$. Now, the lemma easily follows from the following commuting diagram:

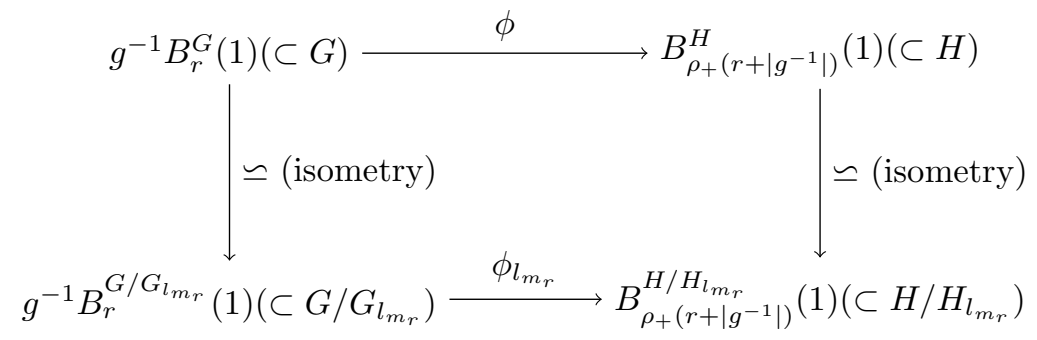

Proof of Proposition 4.1: In the proof of this proposition, we will be using some ideas of the construction of a limiting compact metric space for a 'uniformly totally bounded' sequence of compact metric spaces (see Theorem 7.4.15, p. 274, [5]).

Step 1: By Lemma 4.2, there exists a countable dense collection $S_{n}=\left\{x_{i, n}\right\}_{i=1}^{\infty}$ in each $X_{n}$ such that for every $k$ the first $N_{k}$ points of $S_{n}$, denoted by $S_{n}^{(k)}$, form 
a $(1 / k)$-net in $X_{n}$. Without loss of generality, we assume that $S_{n}^{(k)} \subset S_{n}^{(k+1)}$ for all $k \in \mathbb{N}$. Using Proposition 3.1 and Cantor's diagonal argument, after passing through a subsequence, we obtain that

$$
\left\{x_{i, n}\right\}_{n \in \mathbb{N}} \stackrel{\{n\}_{n \in \mathbb{N}}}{\longrightarrow} x_{i},
$$

for some $x_{i} \in X$ and for all $i \in \mathbb{N}$. Let $S^{(k)}:=\left\{x_{i} \mid i=1, \ldots, N_{k}\right\} \subseteq X$ for all $k$ and $S:=\cup_{k=1}^{\infty} S^{(k)}$. We define $X=\overline{\left\{x_{i}\right\}_{i \in \mathbb{N}}} \subseteq Y$ and $X^{\prime}:=\overline{G \cdot\left\{x_{i}\right\}_{i \in \mathbb{N}}} \subseteq Y$. Since $X$ and $X^{\prime}$ are closed subsets of the compact set $Y$, both $X$ and $X^{\prime}$ are compact, and by definition $X^{\prime}$ is $G$-invariant.

Step 2: In the rest of the paper, we denote the distance functions in $X_{n}$ and $X$ by $d_{X_{n}}$ and $d_{X}$, respectively. The distance $d_{X_{n}}\left(g \cdot x_{i, n}, g^{\prime} \cdot x_{j, n}\right)$ does not exceed 1 , i.e., belongs to a compact interval. Therefore, using 'Cantor's diagonal procedure', we extract a subsequence of $\left\{X_{n}\right\}_{n=1}^{\infty}$ such that after passing through the subsequence $\left\{d_{X_{n}}\left(g \cdot x_{i, n}, g^{\prime} \cdot x_{j, n}\right)\right\}_{n=1}^{\infty}$ converges for all $i, j \in \mathbb{N}$ and for all $g, g^{\prime} \in G$. Moreover, using Lemma 4.3, after passing through another subsequence, say $\left\{n_{m}\right\}_{m \in \mathbb{N}}$, we obtain that

$\left\{g \cdot x_{i, n}\right\}_{n \in \mathbb{N}} \stackrel{\left\{n_{m}\right\}_{m \in \mathbb{N}}}{\longrightarrow} g \cdot x_{i}$ and $\lim _{m \rightarrow \infty} d_{X_{n_{m}}}\left(g \cdot x_{i, n_{m}}, g^{\prime} \cdot x_{j, n_{m}}\right)=d_{X}\left(g \cdot x_{i}, g^{\prime} \cdot x_{j}\right)$, for all $i, j \in \mathbb{N}$ and for all $g, g^{\prime} \in G$.

Step 3: We claim that $S^{(2 k)}$ is a $(1 / k)$-net in $X$ and $X^{\prime}$. Indeed, every set $S_{n}^{(k)}=\left\{x_{i, n} \mid i=1, \ldots, N_{k}\right\}$ is a $(1 / k)$-net in the respective space $X_{n}$. Hence, for every $g \cdot x_{i, n} \in X_{n}$ there is a $j \leq N_{k}$ such that $d_{X_{n}}\left(g \cdot x_{i, n}, x_{j, n}\right) \leq 1 / k$. Since $N_{k}$ does not depend on $n$, for every fixed $g \in G$ and $i \in \mathbb{N}$, there is a $j \leq N_{k}$ such that $d_{X_{n}}\left(g \cdot x_{i, n}, x_{j, n}\right) \leq 1 / k$ for infinitely many indices $n$. Passing to the limit, we obtain that $d_{X}\left(g \cdot x_{i}, x_{j}\right) \leq 1 / k$ for this $j$. Thus, $S^{(2 k)}$ is a $(1 / k)$-net in $X$ and $X^{\prime}$ for all $k$. Moreover, we obtain that $X=X^{\prime}$, which implies that $X$ is $G$-invariant.

Step 4: Since, by Step $2, d_{X}\left(x_{i, n_{m}}, x_{j, n_{m}}\right) \stackrel{m \rightarrow \infty}{\longrightarrow} d_{X}\left(x_{i}, x_{j}\right)$ for all $i, j$, we obtain that $S_{n_{m}}^{(k)}$ converges to $S^{(k)}$ in GH-topology as $m \rightarrow \infty$ for all $k \in \mathbb{N}$. Now, since $S_{n_{m}}$ is dense in $X_{n_{m}}$ and $S$ is dense in $X$, we have $X_{n_{m}}$ converges to $X$ in GH-topology. Hence, we have our proposition.

4.2. Construction of a $G$-invariant measure on $X$. In this section, we construct a $G$-invariant probability measure $\mu$ on $X$. The idea of the construction of this measure uses the concept of Gromov's $\square_{1}$-convergence for metric-measure spaces (17] p. 118).

Proposition 4.4. Let $\left\{\left(X_{n}, d_{X_{n}}\right)\right\}_{n \in \mathbb{N}}$ be the sequence of compact metric spaces which converges to $\left(X, d_{X}\right)$ in Gromov-Hausdorff topology as obtained by Proposition 4.1. We give a G-invariant probability measure $\mu_{n}$ on each $X_{n}$. Then, there exists a $G$-invariant probability measure $\mu$ on $X$.

We prove Proposition 4.4 at the end of this subsection. We prove this proposition by taking the weak* limit of the pushward measure of $\mu_{n}$ on $X$ by a sequence of suitable ' $\xi_{n}$-isometry' from $X_{n}$ to $X$ (possibly after passing through a subsequence), where $\xi_{n} \rightarrow 0$ as $n \rightarrow \infty$. We need 'almost $G$-equivariant' $\xi_{n}$-isometry from $X_{n}$ to $X$ to obtain $G$-invariance of the limiting measure. We prove the existence of such 'almost $G$-equivariant' $\xi_{n}$-isometries in the following proposition.

Proposition 4.5. There exist a subsequence $\left\{n_{k}\right\}_{k \in \mathbb{N}}$ and $\xi_{k}$-isometries $f_{k}: X_{n_{k}} \rightarrow$ $X$ for all $k \in \mathbb{N}$ such that $\sup _{x \in X_{n_{k}}} d_{X_{n_{k}}}\left(g \cdot f_{k}(x), f_{k}(g \cdot x)\right)<\xi_{k}$ for all $g \in B_{k}^{G}\left(1_{G}\right)$, where $\xi_{k} \rightarrow 0$ as $k \rightarrow \infty$.

Before going into the proof of Proposition 4.5, we prove the following lemmas. 
Lemma 4.6. The actions of $G$ on $\left\{X_{n} \mid n \in \mathbb{N}\right\} \cup\{X\}$ are equicontinuous, i.e., $g \in G$ being fixed, for all $\epsilon>0$ there exists a $\delta>0$ such that if $d_{X_{n}}\left(\phi_{n}, \psi_{n}\right)<\epsilon$ (resp. $\left.d_{X}(\phi, \psi)<\epsilon\right)$, then $d_{X_{n}}\left(g \cdot \phi_{n}, g \cdot \psi_{n}\right)<\delta$ (resp. $\left.d_{X}(g \cdot \phi, g \cdot \psi)<\delta\right)$, where $\delta$ only depends on $g$ and $\epsilon$, and $\delta \rightarrow 0$ as $\epsilon \rightarrow 0$.

Proof. We fix $g \in G$ and $\epsilon>0$. Without loss of generality, we assume that $\epsilon<2^{-|g|}$. If $d_{X_{n}}\left(\phi_{n}, \psi_{n}\right)=2^{-R}<\epsilon$, then $d_{X_{n}}\left(g \cdot \phi_{n}, g \cdot \psi_{n}\right) \leq 2^{-(R-|g|)}$. Therefore, the lemma follows for $\left\{X_{n} \mid n \in \mathbb{N}\right\}$ by taking $\delta=\epsilon 2^{|g|}$. The statement for $X$ follows by passing to the limit.

Lemma 4.7. Let $k \in \mathbb{N}$ and $r \in \mathbb{N}$ be two fixed numbers. Then, there exists a subsequence $\left\{n_{m}\right\}_{m \in \mathbb{N}}$ such that $B_{r}^{G}\left(1_{G}\right) \cdot S_{n_{m}}^{(k)}$ converges to $B_{r}^{G}\left(1_{G}\right) \cdot S^{(k)}$ in $G H$ topology and there exists an $\epsilon_{m}$-isometry $f_{m}^{(r, k)}: B_{r}^{G}\left(1_{G}\right) \cdot S_{n_{m}}^{(k)} \rightarrow B_{r}^{G}\left(1_{G}\right) \cdot S^{(k)}$ for all $m$ such that $\left.f_{m}^{(r, k)}\right|_{S_{n m}^{(k)}}$ is $B_{r}^{G}\left(1_{G}\right)$-equivariant, i.e., $f_{m}^{(r, k)}\left(g \cdot x_{i, n_{m}}\right)=g \cdot f_{m}^{(r, k)}\left(x_{i, n_{m}}\right)$ for all $g \in B_{r}^{G}\left(1_{G}\right)$ and for all $i \in\left\{1, \ldots, N_{k}\right\}$, and $\epsilon_{m} \rightarrow 0$ as $m \rightarrow \infty$.

Proof. Using the same argument as given in Step 2 of the proof of Proposition 4.1. after passing through a subsequence, we have

$$
\left\{g \cdot x_{i, n}\right\}_{n \in \mathbb{N}} \stackrel{\{n\}_{n \in \mathbb{N}}}{\longrightarrow} g \cdot x_{i} \text { and } \lim _{n \rightarrow \infty} d_{X_{n}}\left(g \cdot x_{i, n}, g^{\prime} \cdot x_{j, n}\right)=d_{X}\left(g \cdot x_{i}, g^{\prime} \cdot x_{j}\right),
$$

for all $i, j \in\left\{1, \ldots, N_{k}\right\}$ and for all $g, g^{\prime} \in B_{r}^{G}\left(1_{G}\right)$. We fix $g \in G$ and $i \in$ $\left\{1, \ldots, N_{k}\right\}$. If $g \cdot x_{i, n} \in S_{n}^{(k)}$ for infinitely many $n \in \mathbb{N}$, we find a subsequence $\left\{n_{u}\right\}_{u \in \mathbb{N}}$ and $x_{j, n_{u}} \in S_{n_{u}}^{(k)}$ such that $g \cdot x_{i, n_{u}}=x_{j, n_{u}}$ for some $j \in\left\{1, \ldots, N_{k}\right\}$ and for all $u \in \mathbb{N}$. Therefore, we have

$$
\left\{g \cdot x_{i, n_{u}}=x_{j, n_{u}}\right\}_{u \in \mathbb{N}} \stackrel{\left\{n_{u}\right\}_{u \in \mathbb{N}}}{\longrightarrow} g \cdot x_{i}=x_{j} .
$$

If this is not the case, we obtain another subsequence $\left\{n_{v}\right\}_{v \in \mathbb{N}}$ such that $g \cdot x_{i, n_{v}} \notin$ $S_{n_{v}}^{(k)}$ for all $v \in \mathbb{N}$. By Lemma 4.3, we obtain a subsequence $\left\{n_{w}\right\}_{w \in \mathbb{N}}$ of $\left\{n_{v}\right\}_{v \in \mathbb{N}}$ such that

$$
\left\{g \cdot x_{i, n_{w}}\right\}_{w \in \mathbb{N}} \stackrel{\left\{n_{w}\right\}_{w \in \mathbb{N}}}{\longrightarrow} g \cdot x_{i}
$$

Applying the above procedure inductively on the elements of $g \in B_{r}^{G}\left(1_{G}\right)$ and $i \in\left\{1, \ldots, N_{k}\right\}$, we obtain a subsequence $\left\{n_{m}\right\}_{m \in \mathbb{N}}$ such that

$\left\{g \cdot x_{i, n}\right\}_{n \in \mathbb{N}} \stackrel{\left\{n_{m}\right\}_{m \in \mathbb{N}}}{\longrightarrow} g \cdot x_{i}$ and $\lim _{m \rightarrow \infty} d_{X_{n_{m}}}\left(g \cdot x_{i, n_{m}}, g^{\prime} \cdot x_{j, n_{m}}\right)=d_{X}\left(g \cdot x_{i}, g^{\prime} \cdot x_{j}\right)$, for all $g, g^{\prime} \in B_{r}^{G}\left(1_{G}\right)$ and for all $i, j \in\left\{1, \ldots, N_{k}\right\}$. We define $f_{m}^{(r, k)}: B_{r}^{G}\left(1_{G}\right)$. $S_{n_{m}}^{(k)} \rightarrow B_{r}^{G}\left(1_{G}\right) \cdot S^{(k)}$ by mapping $g \cdot x_{i, n_{m}} \mapsto g \cdot x_{i}$. This is crucial to observe that $f_{m}^{(r, k)}$ is a well-defined map. Now, since $d_{X_{n_{m}}}\left(g \cdot x_{i, n_{m}}, g^{\prime} \cdot x_{j, n_{m}}\right) \stackrel{m \rightarrow \infty}{\longrightarrow}$ $d_{X}\left(g \cdot x_{i}, g^{\prime} \cdot x_{j}\right)$, therefore $f_{m}^{(r, k)}$ is an $\epsilon_{m}$-isometry for some $\epsilon_{m}>0$, where $\epsilon_{m} \rightarrow 0$ as $m \rightarrow \infty$.

Proof of Proposition 4.5: Using Lemma 4.7 and Cantor's diagonal procedure, we obtain a subsequence $\left\{n_{k}\right\}_{k \in \mathbb{N}}$ and $\epsilon_{k}$-isometry $f_{k}: B_{k}^{G}\left(1_{G}\right) \cdot S_{n_{k}}^{(k)}\left(\subset X_{n_{k}}\right) \rightarrow$ $B_{k}^{G}\left(1_{G}\right) \cdot S^{(k)}(\subset X)$ such that $\epsilon_{k} \rightarrow 0$ as $k \rightarrow \infty$. Without loss of generality, we assume that $\epsilon_{k}>1 / k$ for all $k \in \mathbb{N}$. Fix $k \in \mathbb{N}$ and $g \in B_{k}\left(1_{G}\right)$. We extend $f_{k}$ on $X_{n_{k}}$ in the following way (we denote the extended map by the same symbol $f_{k}$ ): Let $x \in X_{n_{k}} \backslash B_{k}^{G}\left(1_{G}\right) \cdot S_{n_{k}}^{(k)}$. Then, there exists $x_{i, n_{k}} \in S_{n}^{(k)}$ such that $d_{X_{n_{k}}}\left(x, x_{i, n_{k}}\right)<$ $1 / k$. We define $f_{k}(x):=f_{k}\left(x_{i, n_{k}}\right)$. It is easy to observe that the extended map $f_{k}$ is a $3 \epsilon_{k}$-isometry. Let $\delta_{k}>0$ be the number corresponding to $3 \epsilon_{k}$ obtained by Lemma 4.6. Therefore, $d_{X_{n_{k}}}\left(g \cdot x, g \cdot x_{i, n_{k}}\right)<\delta_{k}$ and $d_{X}\left(g \cdot f_{k}(x), g \cdot f_{k}\left(x_{i, n_{k}}\right)<\delta_{k}\right.$. 
Since $f_{k}$ is a $3 \epsilon_{k}$-isometry, $d_{X}\left(f_{k}(g \cdot x), f_{k}\left(g \cdot x_{i, n_{k}}\right)\right)<3 \epsilon_{k}+\delta_{k}$. Now, we have the following inequality:

$$
\begin{aligned}
d_{X}\left(g \cdot f_{k}(x), f_{k}(g \cdot x)\right) \leq & d_{X}\left(g \cdot f_{k}(x), g \cdot f_{k}\left(x_{i, n_{k}}\right)\right) \\
& +d_{X}\left(g \cdot f_{k}\left(x_{i, n_{k}}\right), f_{k}\left(g \cdot x_{i, n_{k}}\right)\right) \\
& +d_{X}\left(f_{k}\left(g \cdot x_{i, n_{k}}\right), f_{k}(g \cdot x)\right) .
\end{aligned}
$$

By Lemma 4.7, $d_{X}\left(g \cdot f_{k}\left(x_{i, n_{k}}\right), f_{k}\left(g \cdot x_{i, n_{k}}\right)\right)=0$. Therefore, we obtain that

$$
d_{X}\left(g \cdot f_{k}(x), f_{k}(g \cdot x)\right)<3 \epsilon_{k}+2 \delta_{k} .
$$

Hence, we have our proposition by taking $\xi_{k}=3 \epsilon_{k}+2 \delta_{k}$.

Corollary 4.8. Using the notations of Proposition 4.5, we have

$$
d_{X_{n_{k}}}^{\text {Haus }}\left(g \cdot f_{k}^{-1}(A), f_{k}^{-1}(g \cdot A)\right) \leq 2 \xi_{k}
$$

for all $g \in B_{k}^{G}\left(1_{G}\right)$ and for all subsets $A$ of $X$, where $d_{X_{n_{k}}}^{\text {Haus }}$ denotes the Hausdorff distance between two subsets in $X_{n_{k}}$.

Proof. Fix $y \in A$. Let $z=g \cdot x \in g \cdot f_{k}^{-1}(\{y\})$ and $z^{\prime} \in f_{k}^{-1}(\{g \cdot y\})$. Since $f_{k}$ is a $\xi_{k}$-isometry, we have

$$
d_{X_{n_{k}}}\left(z, z^{\prime}\right) \leq \xi_{k}+d_{X}\left(f_{k}(z), f_{k}\left(z^{\prime}\right)\right) .
$$

We observe that $f_{k}(z)=f_{k}(g \cdot x)$ and $f_{k}\left(z^{\prime}\right)=g \cdot f_{k}(x)$. Now, using equation 4.1. we obtain that $d_{X_{n_{k}}}\left(z, z^{\prime}\right) \leq 2 \xi_{k}$. Hence, we have our corollary.

\section{Proof of Proposition 4.4}

By Proposition 4.5, we obtain a subsequence $\left\{n_{k}\right\}_{k \in \mathbb{N}}$ and $\xi_{k}$-isometries $f_{k}$ : $X_{n_{k}} \rightarrow X$ for all $k \in \mathbb{N}$ such that $\sup _{x \in X_{n_{k}}} d_{X_{n_{k}}}\left(g \cdot f_{k}(x), f_{k}(g \cdot x)\right) \leq \xi_{k}$ for all $g \in B_{k}^{G}\left(1_{G}\right)$, where $\xi_{k} \rightarrow 0$ as $k \rightarrow \infty$. For our convenience, we take $\mu_{n}$ as the uniform measure on $X_{n}$. For all $k \in \mathbb{N}$, we define $\widetilde{\mu_{k}}:=f_{k}^{*}\left(\mu_{n_{k}}\right)$, the pushforward measure of $\mu_{n_{k}}$ on $X$ by $f_{k}$. We consider the space of all probability measures on $X$ with weak* topology, which we denote by $\mathcal{P}(X)$. By Banach-Alaoglu theorem, $\mathcal{P}(X)$ is compact in weak* topology. Therefore, there exists a subsequence of $\left\{\widetilde{\mu_{k}}\right\}_{k=1}^{\infty}$ which converges to a probability measure, say $\mu$, on $X$. Without loss of generality, we denote the subsequence by the same notation $\left\{\widetilde{\mu_{k}}\right\}_{k=1}^{\infty}$. We will prove that $\mu$ is $G$-invariant.

It is a known fact from (17, 13. p. 398) that the weak* topology on $\mathcal{P}(X)$ is metrizable and the metric is given by the following Prokhorov metric: $d_{P}^{X}(\lambda, \nu):=$ $\inf \left\{\eta>0 \mid \lambda(A) \leq \nu\left(A^{\eta}\right)+\eta\right.$ and $\left.\nu(A) \leq \lambda\left(A^{\eta}\right)+\eta\right\}$, where $A^{\eta}$ is the $\eta$-neighborhood of $A$. Since the $\sigma$-algebra of $X$ is generated by the countable number of clopen subsets of $X$, it suffices to prove $g \cdot \mu(A)=\mu(A)$ for all clopen subsets $A$ of $X$. We fix a clopen subset $A$ of $X$ and $g \in G$. There exists $k_{0} \in \mathbb{N}$ such that $g \in B_{k}^{G}\left(1_{G}\right)$ for all $k \geq k_{0}$. Since $A$ is a clopen set, $A^{\eta}=A$ for sufficiently small $\eta$. Now, from the definition of $g \cdot \mu$ and $\mu$, we get

$$
(g \cdot \mu)(A)=\mu\left(g^{-1} \cdot A\right)=\lim _{k \rightarrow \infty} \widetilde{\mu_{k}}\left(g^{-1} \cdot A\right)=\lim _{k \rightarrow \infty} \mu_{n_{k}}\left(f_{k}^{-1}\left(\left[g^{-1} \cdot A\right]\right)\right) .
$$

Now, using Corollary 4.8, we have

$$
f_{k}^{-1}\left(g^{-1} \cdot A\right) \subseteq\left[g^{-1} \cdot f_{k}^{-1}(A)\right]^{2 \xi_{k}}
$$

By Lemma 4.6, corresponding to each number $2 \xi_{k}$, we obtain a positive number $\delta_{k}$ tending to zero as $k \rightarrow \infty$ such that

$$
g \cdot\left[g^{-1} \cdot f_{k}^{-1}(A)\right]^{2 \xi_{k}} \subseteq\left[f_{k}^{-1}(A)\right]^{\delta_{k}}
$$

Since $\mu_{n_{k}}$ is $G$-invariant we have

$$
\mu_{n_{k}}\left(\left[g^{-1} \cdot f_{k}^{-1}(A)\right]^{2 \xi_{k}}\right)=\mu_{n_{k}}\left(g \cdot\left[g^{-1} \cdot f_{k}^{-1}(A)\right]^{2 \xi_{k}}\right)
$$


Using the fact that $f_{k}$ is a $\xi_{k}$-isometry, we obtain that

$$
\left[f_{k}^{-1}(A)\right]^{\delta_{k}} \subseteq f_{k}^{-1}\left(A^{\delta_{k}^{\prime}}\right)
$$

where $\delta_{k}^{\prime}:=\xi_{k}+\delta_{k}$ for all $k \in \mathbb{N}$. Now, using the above set-containments and equations (4.3), (4.4), (4.5), (4.6) and (4.7), we obtain that

$$
\mu_{n_{k}}\left(f_{k}^{-1}\left(g^{-1} \cdot A\right)\right) \leq \mu_{n_{k}}\left(f_{k}^{-1}\left(A^{\delta_{k}^{\prime}}\right)\right) .
$$

Finally, using equation (4.2), we get

$$
(g \cdot \mu)(A)=\lim _{k \rightarrow \infty} \mu_{n_{k}}\left(f_{k}^{-1}\left(g^{-1} \cdot A\right)\right) \leq \lim _{k \rightarrow \infty} \mu_{n_{k}}\left(f_{k}^{-1}\left(A^{\delta_{k}^{\prime}}\right)\right)=\mu(A) .
$$

Applying the same argument for $g^{-1}$ and $A$, we obtain our proposition, i.e., $(g$. $\mu)(A)=\mu(A)$ for $g \in G$ and for all Borel subsets $A$ in $X$.

\section{Proof of the main theorem 1.1;}

Part I. We first prove that if the box spaces are coarsely equivalent, then the groups are UME. We prove it in the following steps.

Step 1: Define $Z=H X(\subseteq W)$. Since the $H$-action on $W$ is proper and $X$ is compact, $Z$ is a closed subset of $W$. Clearly, $Z$ is a $G$ and $H$ invariant subset of $W$. Therefore, we can induce the $G$ and $H$ actions from $W$ to $Z$. We also induce the subspace topology from $W$ to $Z$. $Y$ is a compact-open subset of $W$ and $Y \cap Z=X$. So, we obtain that $X_{H}:=X$ is a compact-open fundamental domain of $Z$ under the induced topology.

Step 2: We give the $G$-invariant probability measure $\mu$ on $X$ as constructed in Subsection 4.2 (Proposition 4.4) and extend this measure on $Z$ by translating $\mu$ by the action of $H$. We denote the extended measure by the same symbol $\mu$. Therefore, we obtain a $G$ and $H$-invariant measure $\mu$ on $Z$.

Step 3: Now, we will construct a compact-open fundamental domain $X_{G}$ of $G$. We will follow the construction given in 29] (Theorem 2.1.2, p 131). We define $E_{h}:=\left\{\psi \in Z: \psi\left(1_{G}\right)=h\right\}$ and $K_{h}:=G E_{h}=\{\psi \in Z: \psi$ takes the value $h\}$. Now, we enumerate the elements of $H$ by $h_{0}=1_{H}, h_{1}, h_{2}, \ldots$ and define

$$
X_{G}:=E_{1_{H}} \cup_{i=1}^{\infty}\left(E_{h_{i}} \cap K_{h_{i-1}}^{c} \cap \ldots \cap K_{1_{H}}^{c}\right) .
$$

Since $E_{1_{H}}=X_{H}$, we have $X_{H} \subset X_{G}$. We refer the reader 29] (Theorem 2.1.2, p 131) for the proof of the fact that $X_{G}$ is a compact-open fundamenatal domain of $G$ in $Z$.

Step 4: Now, it remains to show that $g$-translate of $X_{H}$ can be covered by finitely many $H$-translates of $X_{H}$ for $g \in G$ and $h$-translate of $X_{G}$ can be covered by finitely many $G$-translates of $X_{G}$ for $h \in H$. This easily follows from the fact that $X_{G}$ and $X_{H}$ are compact-open in $Z$.

By Lemma A.1 in 7, the composition of two UME's is a UME. Since $H \times M$ and $H$ are commensurable, therefore there is a UME between $G$ and $H$.

Part II. In this part, we prove that if there is a coarse embedding of one box space into another box space, then there is a UME-embedding of the first group into the second one. Since the proof of this part will be almost same as Part I, we will give a brief sketch of it. We will be using same notations from the previous part. Here, we consider $Y$ as $\left\{f: G \rightarrow H \mid f\right.$ is a $\left(\rho_{+}, \rho_{-}\right)-$ coarse embedding and $\left.f\left(1_{G}\right)=1_{H}\right\}$ and $X_{n}$ as $\left\{f: G / G_{n} \rightarrow H / H_{n} \mid f\right.$ is a $\left(\rho_{+}, \rho_{-}\right)$- coarse embedding and $\left.f\left(1_{G / G_{n}}\right)=1_{H / H_{n}}\right\}$. We can use a similar result like Proposition 4.1 to show that there exists a subsequence of $\left\{X_{n}\right\}_{n \in \mathbb{N}}$ which 
converges to a $G$-invariant compact subspace $X$ of $Y$ in Gromov-Hausdorff topology. Similarly, we can give a $G$-invariant measure $\mu$ on $X$. We define the coupling space as $Z=H X$ with $X_{H}:=X$ as the fundamental domain for $H$. Since $X_{H}$ is compact-open in $Z$, each $g$-translate of $X_{H}$ can be covered by finitely many $H$ translates of $X_{H}$ for all $g \in G$. Again using Lemma A.1 in [7], we conclude that there is a UME-embedding from $G$ into $H$.

Remark 4.9. With the assumptions of our theorem, we obtain from the proof that there exists a UME between $G$ and $H \times M$ (resp. UME-embedding from $G$ into $H \times M)$ with $X_{H \times M} \subseteq X_{G}$ for some finite group $M$.

Remark 4.10. The theorem 1.1 can be generalized in the setting of amenable 'marked groups' in the following sense: Let $\left\{G_{n}\right\}_{n \in \mathbb{N}}$ and $\left\{H_{n}\right\}_{n \in \mathbb{N}}$ be two sequences of finitely generated amenable marked groups which converge to the finitely generated groups $G$ and $H$ respectively. Suppose there exists $\left(\rho_{+}, \rho_{-}, c\right)$-coarse equivalence from $G_{n}$ to $H_{n}$ for all $n$, where $\left(\rho_{+}, \rho_{-}, c\right)$ is independent of $n$. Then, $G$ and $H$ are uniformly measured equivalent (see [12]).

\section{Applications}

The coarse embedding has been mainly studied for embedding locally finite countable metric spaces with bounded geometry inside a Hilbert space in the context of Baum-Connes conjecture. It arises a natural question of studying the coarse embedding inside the category of countable locally finite metric spaces with bounded geometry. The coarse-equivalence or quasi-isometry has been extensibly studied in geometric group theory among the Cayley graphs of finitely generated groups. But, we do not know much about the coarse embedding of a Cayley graph inside another Cayley graph. Shalom has discussed some of the results in this direction in 29.

In this section, we discuss some questions of coarse equivalence and coarse embedding among box spaces. A. Naor and M. Mendel [27] first construct two expander sequences so that there exists no coarse embedding of one expander sequence into the other one. In their examples, one expander sequence has unbounded girth and another one has many short cycles. Later, D. Hume has given existence of a continuum of expander sequences with unbounded girth 21. Khukhro-Valette obtains 23 examples of another such family with bounded girth: one family is a box space of $S L_{n}(\mathbb{Z})(n \geq 3)$, a group with Property $(\mathrm{T})$, and another family is a box space of $S L_{2}(\mathbb{Z}[\sqrt{p}])$ ( $p$ is a prime), a group with Haagerup property and Property $\tau$. Using part (ii) of Corollary 1.2 we prove that there is no coarse embedding of the box spaces of $S L_{n}(\mathbb{Z})$ into the box spaces of $S L_{m}(\mathbb{Z})$, where $n>m$ and $n, m \geq 3$. Some considerations of non coarsely equivalent box spaces can be found in [2].

\section{Proof of Corollary 1.2;}

Proof of (i): Suppose there is a coarse embedding of a box space of $G$ into a box space of $H$. Then, by Theorem 1.1, there exists a UME-embedding of $G$ into $H$ with a compact fundamental domain $X_{H}$ of $G$. Suppose, $H$ has a metrically proper affine action $\alpha$ on some $L^{p}(Y), 1 \leq p \leq \infty$. We define the induced representation on $L^{p}\left(X_{H} ; L^{p}(Y)\right) \equiv L^{p}\left(X_{H} \times Y\right)$ with the induced cocycle as $t^{\prime}(g)(x):=t(c(g, x))$ where $t$ is the 1-cocyle associated to the affine action $\alpha$ and $c: G \times X_{H} \rightarrow H$ is the cocycle associated with the $(G, H)$-coupling space $(\Omega, \mu)$. We have

$$
\left\|t^{\prime}(g)\right\|=\left(\int_{X_{G}}\|t(c(g, x))\|^{p} d \mu(x)\right)^{1 / p}<\infty
$$

Now, we also have $\rho_{-}(|g|) \leq|c(g, x)| \leq \rho_{+}(|g|)$ for all $x \in X_{H}$. Therefore, $\left\|t^{\prime}(g)\right\| \geq$ $\left(\mu\left(X_{H}\right)\right)^{1 / p}\left\|t\left(\rho_{-}(g)\right)\right\|$. Since $\rho_{-}$is a proper function and $t$ is a proper 1-cocyle, $t^{\prime}$ is a proper 1-cocyle too. Hence, we have our corollary. 
Proof of (ii): By Theorem 1.1 and Remark 4.9, there exist a finite group $M$ and a UME-embedding of $G$ into $H \times M$ with a compact fundamental domain $X_{H \times M}$ of $H \times M$, which is inside the fundamental domain $X_{G}$ of $G$. Since $c d_{R}(H \times M)=$ $c d_{R} H$, without loss of generality, we can replace $H \times M$ by $H$. Now, we can use the same argument as given in the proof of Theorem 1.5 in [29] to conclude that $c d_{R} G \leq c d_{R} H$.

5.1. Distinguishing box spaces up to coarse embedding. We are now ready to discuss the questions of coarse embedding among the box spaces of the following classes of groups. Some of the already known examples can be deduced from Corollary 1.2.

I. $S L_{n}(\mathbb{Z})$ and $S L_{m}(\mathbb{Z})$, where $n>m$ and $n, m \geq 3$ :

Using part (ii) of Corollary 1.2 , we get that if there exists a coarse embedding of a box space of $S L_{n}(\mathbb{Z})$ inside $S L_{m}(\mathbb{Z})$, then $c d_{R} S L_{n}(\mathbb{Z}) \leq c d_{R} S L_{m}(\mathbb{Z})$. But, by a result of Borel-Serre [8, we get explicit values of cohomological dimension of these groups with coeffcients in the $\operatorname{ring} \mathbb{Q}: c d_{\mathbb{Q}} S L_{n}(\mathbb{Z})=\operatorname{dim} N$, where $N$ is set the upper triangular unipotent matrices which appears in the Iwasawa decomposition of $S L_{n}(\mathbb{R})$. Therefore, there exists no coarse embedding of the expanders obtained from $S L_{n}(\mathbb{Z})$ into the expanders obtained from $S L_{m}(\mathbb{Z})$, where $n>m$.

This also can be proved in the following way. By a remark, given in Subsection 6.1 of [29], if there exists a coarse embedding of $S L_{n}(\mathbb{Z})$ into $S L_{m}(\mathbb{Z}), c d_{\mathbb{Q}} S L_{n}(\mathbb{Z}) \leq$ $c d_{\mathbb{Q}} S L_{m}(\mathbb{Z})$. We recall the argument briefly: The intersection of $N$ with $S L_{n}(\mathbb{Z})$, denoted by $N(\mathbb{Z})$, is coarsely embedded in $S L_{n}(\mathbb{Z})$. Now, by Theorem 1.5 in [29], if $\Lambda$ is amenable and if $\Lambda$ coarsely embeds in $\Gamma$, then $c d_{\mathbb{Q}} \Lambda \leq c d_{\mathbb{Q}} \Gamma$. However, $c d_{\mathbb{Q}} N(\mathbb{Z})=c d_{\mathbb{Q}} S L_{n}(\mathbb{Z})=\operatorname{dim} N$. Now, by using Proposition 3.1] we have the above result.

II. Groups with Property T and groups with Haagerup Property: The groups with Property $(\mathrm{T})$ have the property that any affine isometric action of these groups on a Hilbert space has a fixed point. But, the groups with Haagerup property have metrically proper affine isometric action on a Hilbert space. Therefore, the part (i) of Corollary 1.2 easily distinguishes the box spaces of these two groups up to coarse embedding. This also can be proved by using the following result : a finitely generated, residually finite group has Haagerup property if and only if one (or equivalently, all) of its box spaces admits a fibred coarse embedding into a Hilbert space [10. This argument has been used by Khukhro-Valette in 23] to show that there exists no coarse embedding of the box spaces of $S L_{n}(\mathbb{Z})$, where $n \geq 3$, into the box spaces of $S L_{2}(\mathbb{Z}[\sqrt{p}])$, where $p$ is a prime.

III. Lattices of $S L_{n}(\mathbb{R})(n \geq 3)$ and a finite product of hyperbolic groups: By [6], the lattices in $S L_{n}(\mathbb{R})$ have Property $F_{L^{p}}$, where $1<p<\infty$, i.e., any affine isometric action of these groups on an $L^{p}$-space $(1<p<\infty)$ has a fixed point. On the other hand, by [32, hyperbolic groups admit a metrically proper affine isometric action on an $l^{p}$-space for some $2 \leq p<\infty$. Therefore, by part (ii), there is no coarse embedding of the box spaces of lattices in $S L_{n}(\mathbb{R})$ inside the box spaces of a finite product of hyperbolic groups. This also can be argued by using a result about 'fibred coarse embedding inside an $L^{p}$-space', where $1 \leq p \leq \infty$ : a finitely generated, residually finite group has a proper affine action on an $L^{p}$-space if and only if one (or equivalently, all) of its box spaces admits a fibred coarse embedding into an $L^{p}$-space 11. In particular, since the lattices in $\operatorname{Sp}(n, 1)(n \geq 2)$ are hyperbolic groups with Property $(\mathrm{T})$, using our result we obtain that there is no coarse embedding of the expander sequences coming from $S L_{n}(\mathbb{Z})(n \geq 3)$ into the expanders coming from the lattices of $S p(n, 1)(n \geq 2)$. 
5.2. Distinguishing box spaces up to coarse equivalence: In this subsection, we show a countable class of coarsely equivalent groups such that the box spaces of two such groups are not coarsely equivalent. The examples of residually finite groups which are quasi-isometric but not measured equivalent will serve our purpose. The following class of examples has been suggested by D. Gaboriau: Let $\Gamma_{p, q, r}:=$ $\left(F_{p} \times F_{q}\right) * F_{r}$, where $F_{p}, F_{q}$ and $F_{r}$ are free groups with $p, q$ and $r$ generators, respectively, and $p, q, r \geq 2$.

Corollary 5.1. There exists a countable class of residually finite groups from the collection

$$
\left\{\Gamma_{p, q, r} \mid(p, q, r) \in \mathbb{N} \times \mathbb{N} \times \mathbb{N}\right\}
$$

where any two groups are coarsely equivalent but any of their box spaces are not coarsely equivalent.

Proof. Since all free groups with at least two generators are commensurable, the groups $\Gamma_{p, q, r}$ for different $(p, q, r)$ are quasi-isometric. Using Properties 1.5 and Example 1.6 of [15] (p. 12,13), we compute the first and second $l^{2}$-betti numbers of this group: $\beta_{1}^{(2)}\left[\left(F_{p} \times F_{q}\right) * F_{r}\right]=r$ and $\beta_{2}^{(2)}\left[\left(F_{p} \times F_{q}\right) * F_{r}\right]=(p-1)(q-1)$. By Corollary 1.3. the betti numbers of $G$ and $H$ must be proportional. Therefore, we can obtain a countable class of residually finite groups from $\left\{\Gamma_{p, q, r} \mid(p, q, r) \in\right.$ $\mathbb{N} \times \mathbb{N} \times \mathbb{N}\}$ which are not mutually measured equivalent. Now, using Theorem 1.1. we obtain that any two box spaces of two diffrent groups from the abovementioned class are not coarsely equivalent.

Remark 5.2. The above corollary shows that Theorem 1.1 is stronger than Proposition 3.1 i.e., there are examples of groups whose box spaces can not be distinguished up to coarse-equivalence by 3.1 but can be distinguished by 1.1 .

\section{Some QUeSTIONS}

The author does not know 'whether there exist two residually finite groups $\Gamma$ and $\Lambda$ such that there exists a coarse embedding of $\Gamma$ into $\Lambda$ but there exists no UMEembedding of $\Gamma$ into $\Lambda$ '. If such examples exist, this will assure that Theorem 1.1 is stronger than Proposition 3.1 in terms of distinguishing box spaces up to coarse embedding. We suspect that this might be a possible example: We know from above that there is no coarse embedding of the box spaces of a lattice inside a simple Lie group of higher rank, for example $S L_{n}(\mathbb{Z})(n \geq 3)$, into the box spaces of a finite product of hyperbolic groups. But, it is not known to the author whether there exists a coarse embedding of $S L_{n}(\mathbb{Z})(n \geq 3)$ into a finite product of hyperbolic groups or into a finite product of 3-regular trees. A similar question was asked by Cornulier in 9] (Question 1.12, p. 5).

The author would also like to know whether the converse of the main theorem 1.1 is true, i.e., is it true that if two residually finite groups are uniform measured equivalent, then there exist box spaces of each of them which are coarsely equivalent?

\section{REFERENCES}

[1] S. Arnt, Fibred coarse embeddability of box spaces and proper isometric affine actions on $L^{p}$ spaces. Preprint 2015. arXiv:1508.05033

[2] G. Arzhantseva and E. Guentner, Coarse non-amenability and covers with small eigenvalues, Mathematische Annalen, 354(3) (2012), 863-870.

[3] G. Arzhantseva, E. Guentner, and J. Spakula, Coarse non-amenability and coarse embeddings, Geom. Funct. Anal. 22 (2012), no. 1, 22-36.

[4] G. Arzhanseva and R. Tessera, Relative expanders. Geometric and Functional Analysis April 2015, Volume 25, Issue 2, pp 317-341. 
[5] D. Burago, Y. Burago, S. Ivanov, A course in Metric Geometry. AMS Graduate Studies in Mathematics, Volume 33, 2001.

[6] U Bader, A Furman, T Gelander, N Monod, Property (T) and rigidity for actions on Banach spaces. Acta Mathematica, March 2007, Volume 198, Issue 1, pp 57-105.

[7] U. Bader, A. Furman, R. Sauer. Integrable measure equivalence and rigidity of hyperbolic lattices. Inventiones mathematicae, November 2013, Volume 194, Issue 2, pp 313-379.

[8] A. Borel and J.P. Serre, Corners and arithmetic groups. Comment. Math. Helv., 48 (1973), 436-491.

[9] Y. de Cornulier, Dimension of asymptotic cones of Lie groups. J. Topology 1(2), 343-361, 2008.

[10] X. Chen, Q. Wang and X. Wang. Characterization of the Haagerup property by fibred coarse embedding into Hilbert space Bull. Lond. Math. Soc. 45 (2013), no. 5, 1091-1099.

[11] X. Chen, Q. Wang, G. Yu, The maximal coarse Baum-Connes conjecture for spaces which admit a fibred coarse embedding into Hilbert space. Advances in Mathematics, Volume 249, 20 December 2013, Pages 88-130.

[12] K. Das, PhD Thesis. To appear.

[13] D. Daley and D. Vere-Jones, An Introduction to the Theory of Point Processes. Probability and its Applications. Springer-Verlag, New York, 2003.

[14] G. Elek, The combinatorial cost. l'Enseignement Math. 53, 225-236 (2007).

[15] D. Gaboriau, Invariants $l^{2}$ de relations d'équivalence et de groupes, Publ. Math. Inst. Hautes Etudes Sci. 95 (2002), 93-150.

[16] M. Gromov. Asymptotic invariants of infinite groups. In G. Niblo and M. Roller (Eds.), Geometric group theory II, number 182 in LMS lecture notes. Camb. Univ. Press, 1993.

[17] M. Gromov, Metric Structure for Riemannian and Non-Riemannian Spaces. Modern Birkauser Classics, 1999.

[18] M. Gromov, Spaces and questions, (Tel Aviv, 1999), Geom. Funct. Anal. 2000, Special Volume, Part I, 118-161.

[19] E. Guentner and J. Kaminker, Geometric and Analytic Properties of Groups. Noncommutative Geometry, Volume 1831 of the series Lecture Notes in Mathematics pp 253-262, December 2003.

[20] P. de la Harpe, Topics in geometric group theory. University of Chicago Press (2000).

[21] D. Hume, A continuum of expanders. Preprint 2015. arXiv:1410.0246

[22] A. Khukhro, Box spaces, group extensions and coarse embeddings into Hilbert space, Journal of Functional Analysis 263, 2012.

[23] A. Khukhro and A. Valette, Expanders and box spaces. Preprint 2015. arXiv:1509.01394.

[24] A. Lubotzky and A. Zuk, On property $(\tau)$, preliminary version available online, 2003.

[25] G. Margulis, Explicit group theoretic constructions of combinatorial schemes and their applications in the construction of expanders and concentrators, Problems Inform. Transmission 24, 1988.

[26] J. Matoušek, On embedding expanders into $l^{p}$ spaces, Israel J. Math., 102 (1997), 189197.

[27] M. Mendel and A. Naor, Nonlinear spectral calculus and super-expanders, Publications mathématiques de l'IHÉS 119, issue 1 (2014), pages 1-95.

[28] J. R. Munkres, Topology. Second Edition, Prentice Hall, 2000.

[29] Y. Shalom. Harmonic analysis, cohomology, and the large scale geometry of amenable groups. Acta Mathematica 193 (2004), 119-185.

[30] R. Willett, G. Yu. Geometric property (T). Chinese Annals of Mathematics, Series B September 2014, Volume 35, Issue 5, pp 761-800.

[31] G. Yu, The coarse Baum-Connes conjecture for spaces which admit a uniform embedding into Hilbert space. Invent. Math., 139(1):201-240, 2000.

[32] G. Yu, Hyperbolic groups admit proper affine isometric action on $l^{p}$-spaces. Geometric and Functional Analysis, Vol. 15, 5 (2005) 1114-1151.

UMPA UMR 5669, ENS Lyon, 69364 Lyon CEDEX 7, FRANCE

E-mail address: kajal.das@ens-lyon.fr 